\title{
Inadvertent arterial insertion of a central venous catheter
}

Hong Kong Med J 2018;24:427.e1-2

Victor WT Chan *, KW Shek

DOI: $10.12809 / \mathrm{hkmj} 176866$

Central venous catheterisation is a common procedure that allows venous access for delivering medications, infusing fluids or blood products, and monitoring volume status. Traditionally, anatomical landmarks of the sternocleidomastoid muscle provide a pathway to catheterise the internal jugular vein (IJV). However, inadvertent arterial puncture is a risk. Currently, ultrasound guidance by experienced operators is recommended for reducing the risk of mechanical complications during central venous catheter (CVC) insertion. ${ }^{1}$

For venous access via the neck, common carotid and subclavian artery injuries have been reported..$^{2}$ The risk of artery injury is about $0.5 \%$, and practice should be reviewed if the risk exceeds $1 \%$. Other known mechanical complications include haematoma formation, haemothorax, and pneumothorax.

A 55-year-old woman with end-stage renal failure on continuous ambulatory peritoneal dialysis was admitted to our hospital for fever and abdominal pain. The clinical diagnosis of continuous ambulatory peritoneal dialysis peritonitis was made, and the peritoneal dialysis catheter was removed. Bedside insertion of a CVC was selected for temporary haemodialysis. The CVC insertion was initially attempted via the right IJV, unsuccessfully. The CVC was subsequently inserted via the left IJV. The procedure was performed under ultrasound

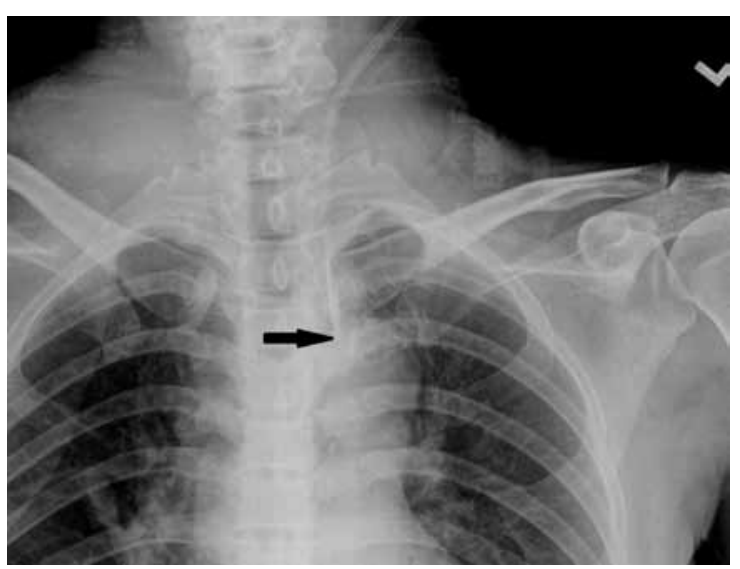

FIG I. Chest radiograph after central venous catheter insertion showing an abnormal vertical course of the catheter with suspected malposition. The catheter tip is seen close to the aortic arch (arrow) guidance using the Seldinger technique; however, inadvertent arterial puncture was not recognised, and the procedure was continued.

After CVC insertion, a chest radiograph was taken, showing an abnormal vertical course of the catheter with suspected malposition (Fig 1). Urgent contrast computed tomographic angiogram (Figs 2 and 3) revealed that the catheter had been inserted via the left IJV, subsequently exiting posteromedially, entering the left vertebral artery, and harbouring at the origin of the left subclavian artery. Computed tomographic angiogram also showed abnormal contrast pooling over the right neck suggestive of a pseudoaneurysm formation from the right subclavian artery.

The opinion of a vascular surgeon was sought and the catheter was removed under general

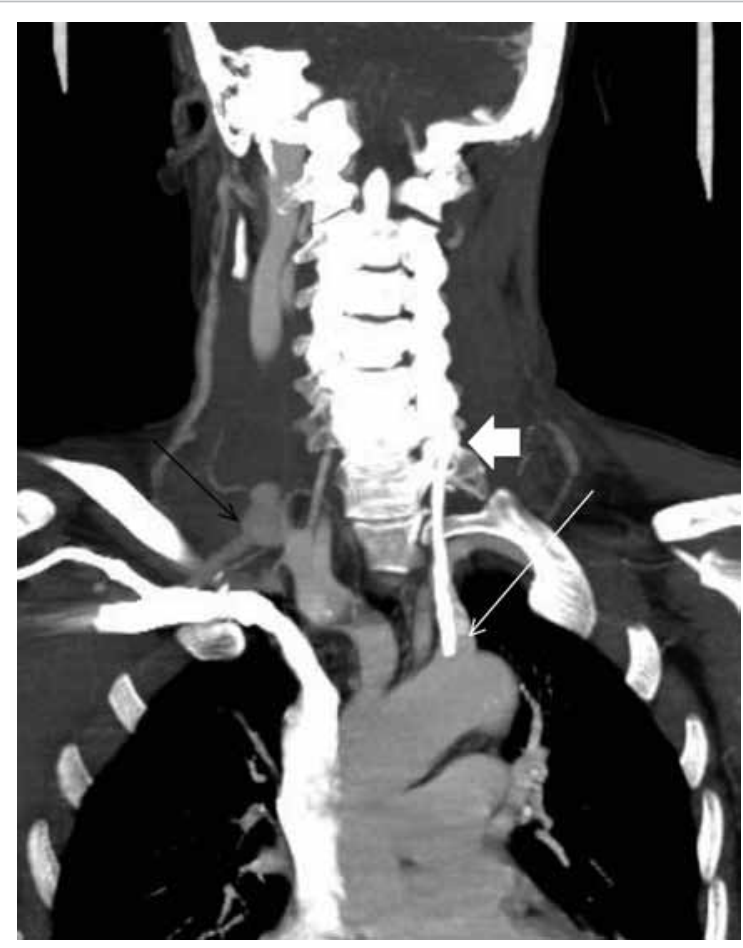

FIG 2. Contrast computed tomographic angiogram with maximum intensity projection showing the central venous catheter (thick white arrow) with a vertical course and punctured the left vertebral artery. The catheter tip is seen at the origin of the left subclavian artery (thin white arrow). Over the right neck, a contrast pooling pseudoaneurysm (black arrow) connected to the right subclavian artery was identified 


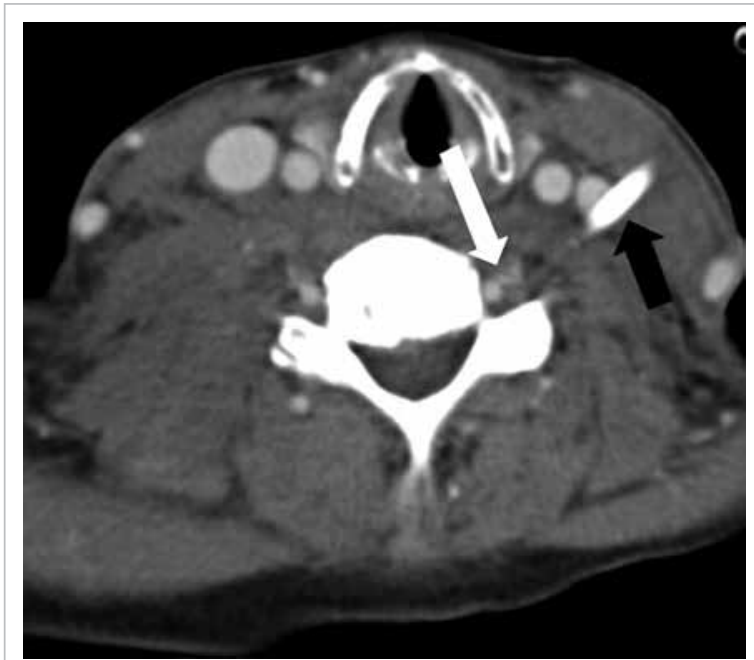

FIG 3. Contrast computed tomographic angiogram showing the central venous catheter puncturing through the left internal jugular vein, exiting medially (black arrow). The catheter enters the left vertebral artery (white arrow) located posteromedially

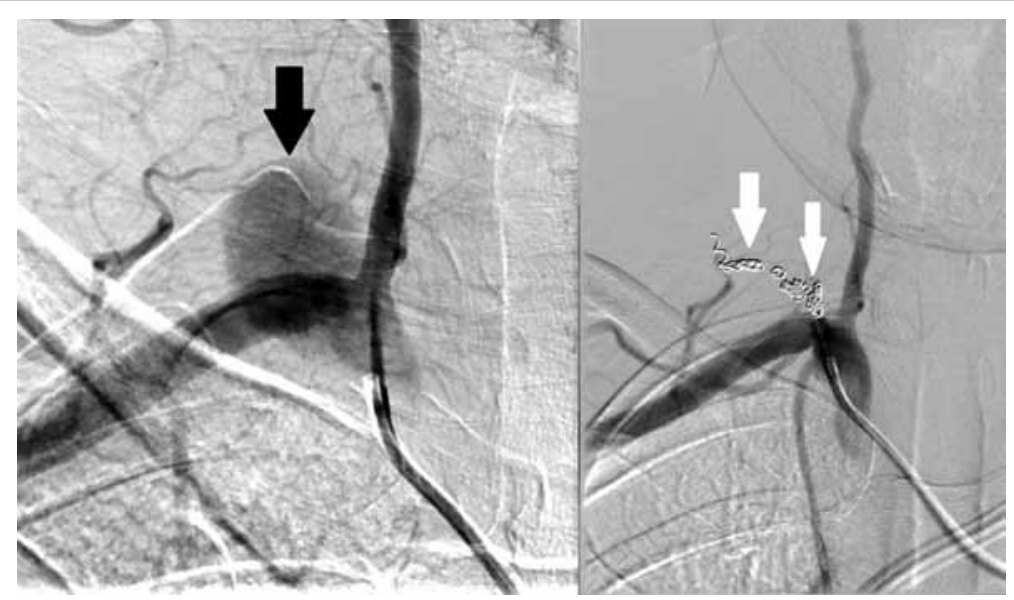

FIG 4. Right subclavian angiogram showing a pseudoaneurysm (black arrow) arising from the right thyrocervical trunk, which was successfully embolised with coils (white arrows)
Postoperatively, the patient progressed well, with her peritonitis controlled by intravenous antibiotics. A new $\mathrm{CVC}$ for temporary haemodialysis was inserted via her right IJV by interventional radiologists under fluoroscopic and real-time ultrasound guidance.

This case concurs with a previous report that the incidence of mechanical complications after multiple attempts is higher than after one attempt. ${ }^{2}$ Real-time ultrasound guided venepuncture of the IJV has a higher first insertion attempt success rate, and decreased rate of arterial puncture as compared with the anatomic landmark approach; this technique is currently recommended by the Association of Anaesthetists of Great Britain and Ireland for all CVC insertions. ${ }^{3}$ Credentialing of ultrasound-guided CVC insertion should be advocated in Hong Kong, with adequate training provided by accredited trainers.

\section{Author contributions}

All authors have made substantial contributions to the concept of this pictorial medicine; acquisition and interpretation of data, drafting of the article, and critical revision for important intellectual content.

\section{Funding/support}

This research received no specific grant from any funding agency in the public, commercial, or not-for-profit sectors.

\section{Declaration}

All authors have disclosed no conflicts of interest. All authors had full access to the data, contributed to the study, approved the final version for publication, and take responsibility for its accuracy and integrity.

VWT Chan *, MB, BS, FRCR

KW Shek, MB, BS, FRCR

Department of Radiology and Imaging, Queen Elizabeth Hospital, Jordan, Hong Kong

* Corresponding author: chanwaitat@gmail.com

\section{References}

1. McGee DC, Gould MK. Preventing complications of central venous catheterization. $N$ Engl J Med 2003;348:1123-33.

2. Schummer W, Schummer C, Rose N, Niesen WD, Sakka SG. Mechanical complications and malpositions of central venous cannulations by experienced operators. A prospective study of 1794 catheterizations in critically ill patients. Intensive Care Med 2007;33:1055-9.

3. Bodenham A, Babu S, Bennett J, et al. Association of Anaesthetists of Great Britain and Ireland: Safe Vascular Access 2016. Anaesthesia 2016;71:573-85. 